\title{
Criminologie
}

\section{La consommation de substances psycho-actives chez les femmes détenues au Québec}

\section{Serge Brochu, Louise Biron et Lyne Desjardins}

Volume 29, numéro 1, printemps 1996

Intervenir auprès des délinquants

URI : https://id.erudit.org/iderudit/017384ar

DOI : https://doi.org/10.7202/017384ar

Aller au sommaire du numéro

Éditeur(s)

Les Presses de l'Université de Montréal

ISSN

0316-0041 (imprimé)

1492-1367 (numérique)

Découvrir la revue

Citer cet article

Brochu, S., Biron, L. \& Desjardins, L. (1996). La consommation de substances psycho-actives chez les femmes détenues au Québec. Criminologie, 29(1),

121-139. https://doi.org/10.7202/017384ar
Résumé de l'article

Compared to male offenders, very few studies have been published on drug use by female offenders. This paper, base on the interviews of 94 female offenders incarcerated aim to enlighten this obscure situation. Results indicate that drug use by female inmates is very important and, in some way problematic. It is not to say that their drug use cause crimes, but their drug problem need to be addressed thoughtfully. 
CONSOMMATION DE SUBSTANCES PSYCHO-ACTIVES

CHEZ LES FEMMES DÉTENUES AU QUÉBEC ${ }^{1} 2$

Serge Brochu ${ }^{3}$

Louise Biron ${ }^{4}$

Lyne Desjardins ${ }^{5}$

Compared to male offenders, very few studies have been published on drug use by female offenders. This paper, base on the interviews of 94 female offenders incarcerated aim to enlighten this obscure situation. Results indicate that drug use by female inmates is very important and, in some way problematic. It is not to say that their drug use cause crimes, but their drug problem need to be addressed thoughtfully.

L'abus de drogues existe depuis des millénaires et va sûrement persister au fil des siècles. Cependant, il peut prendre différentes formes et attirer divers types de ṕersonnes. Ainsi, depuis le début des années 1960, un observateur attentif peut facilement constater certains changements. Alors que la consommation excessive de substances psycho-actives concernait des groupes très spécifiques, ce phénomène se retrouve aujourd'hui dans toutes les classes de la société. De même, la nature et la force des produits se sont modifiées avec le temps.

De plus en plus, dans l'imagerie populaire comme dans certains exposés scientifiques, l'usage et l'abus de certaines substances psycho-actives sont associés à des comportements déviants. D'autres discours vont encore plus loin en les associant à des comportements criminels. À écouter les reportages dans les médias, on est en droit de croire que la relation entre drogues illicites et criminalité va de soi en Amérique du Nord. Il s'agit certainement d'une source d'inquiétude considérable pour les parents qui entendent ces reportages et qui croient alors que la consommation de drogues illicites de leurs adolescents les mènera directement à la criminalité.

1. Cette étude a été réalisée grâce à une subvention du Conseil de recherche en sciences humaines du Canada.

2. Les auteurs désirent remercier les deux évaluateurs anonymes pour leurs judicieuses remarques.

3. Professeur agrégé à l'École de criminologie et chercheur au Groupe de recherche et d'intervention sur les substances psycho-actives, Québec (RISQ), Centre international de criminologie comparée, Université de Montréal, C.P. 6128, Succ. Centre Ville, Montréal, Qc, H3C 3J7.

4. Professeure agrégée à l'École de criminologie et chercheure associée au Centre international de criminologie comparée, Université de Montréal.

5. Agente de recherche, Groupe de recherche en intervention sur les substances psycho-actives, Québec (RISQ), Centre international de criminologie comparée, Université de Montréal. 
La consommation de drogues et, à plus juste titre, la toxicomanie des personnes judiciarisées préoccupe les intervenants ainsi que la direction des établissements de détention qui doivent contribuer à assurer la protection de la société, mais également offrir des services de santé adéquats aux personnes qui sont sous leur juridiction. En effet, la dernière décennie nous a inondés de rapports de recherche en provenance des États-unis indiquant l'importance des problèmes de consommation de drogues chez les personnes judiciarisées et le lien entre cette consommation et une récidive probable (voir Brochu, 1995, pour une recension exhaustive et une analyse de ces écrits scientifiques). Bien que ces rapports ne distinguent pas toujours adéquatement la simple consommation de la toxicomanie, ils ont eu pour effet d'alerter les intervenants.

Cet article a pour but de présenter les résultats d'une étude épidémiologique concernant la consommation de substances psycho-actives des femmes détenues au Québec. L'obtention de données québécoises permet ainsi d'adapter les programmes aux besoins des gens d'ici au lieu de se contenter d'extrapoler les résultats des études américaines. Pour bien analyser ces chiffres et comprendre leur importance, il devient toutefois nécessaire de connaître la consommation de l'ensemble des femmes québécoises.

\section{PROBLÉMATIQUE}

\section{A. Prévalence de consommation de substances psycho-actives au Québec}

En mars 1989, Statistique Canada a procédé à des entrevues téléphoniques au nom de Santé et Bien-être Canada en vue de réaliser une enquête nationale sur l'alcool et les autres drogues. L'échantillon était constitué, entre autres, de 1808 Québécoises et Québécois de 15 ans et plus qui ont rapporté leur consommation de substances psycho-actives pour des périodes préétablies. Il s'avère donc possible d'analyser les résultats de cette enquête nationale pour le Québec (Santé et Bien-être social Canada, 1990).

L'usage d'alcool au Québec est surtout une «affaire» d'hommes. Le nombre moyen de consommations par semaine reflète bien cette constatation. Alors que les Québécoises disent consommer en moyenne 1,7 verre, leur contrepartie masculine signale une consommation moyenne de 5,7 verres (Santé et Bien-être social Canada, 1990).

Par ailleurs, l'usage licite de médicaments est surtout une «affaire » de femmes. Ainsi, deux fois plus de Québécoises que de Québécois rapportent avoir absorbé des somnifères (respectivement $6,1 \%$ et $2,9 \%$ ) ou des tranquillisants (respectivement $5,9 \%$ et $2,5 \%$ ) au cours des trente jours qui ont précédé l'étude. Le Québec constitue la province où l'on retrouve le plus 
baut pourcentage de consommateurs de somnifères et de -tranquillisants au Canada (somnifères: Québec $=4,5 \%$; Canada $=3,6 \%$; tranquillisants : Québec $=4,3 \%$; Canada $=3,1 \%$ ).

L'enquête canadienne a également permis de recueillir des données concernant l'utilisation de drogues illicites. Ainsi, 15,6\% des Québécoises (contre $27,4 \%$ des Québécois) ont consommé, à un moment de leur vie, de la marijuana ou đu haschisch et près đe quatre Québécoises sur cent $(3,8 \%)$ en ont fait usage dans les 12 mois qui ont précédé l'enquête (hommes : $9,2 \%$ ). Lorsqu'il s'agit de cocaïne, les pourcentages baissent considérablement. Ainsi, 3,0\% des Québécoises en ont pris au cours de leur vie (hommes : $5,1 \%$ ) et $2,0 \%$ en ont consommé au cours de l'année qui précédait l'enquête. Les autres drogues étudiées (LSD, speed et héroïne) n'ont été utilisées que par une très faible proportion de Québécois. Les responsables de l'étude avertissent d'ailleurs les lecteurs que la forte variabilité des échantillons rend ces dernières données difficiles à analyser.

Une autre étude épidémiologique majeure avait été réalisée deux années auparavant au Québec. L'Enquête Santé Québec a effectivement été effectuée en 1987 auprès d'un échantillonnage de familles de toutes les régions socio-sanitaires du Québec, à l'exclusion du Nouveau Québec et des réserves indiennes. 13885 familles ont été visitées et 11323 ont accepté de répondre aux questions posées. Un total de 19724 personnes de 15 ans et plus a complété leur questionnaire (Ministère de la Santé et des Services sociaux, 1988).

En ce qui conceme l'alcool, il fut déterminé que parmi la population féminine, $20,3 \%$ s'abstenaient de toute consommation, $49,7 \%$ étaient des buveuses habituelles $^{6}, 25,1 \%$ buvaient occasionnellement ${ }^{7}$ et $4,9 \%$ étaient d'anciennes buveuses. Dans l'ensemble, les femmes consomment moins que les hommes. Tant chez les uns que chez les autres, ce sont les moins de 45 ans qui représentent les grands utilisateurs ${ }^{8} \mathrm{~d}$ 'alcool.

L'étude québécoise de 1987 est confirmée par les résultats de l'étude de Santé et Bien-être social Canada (1990) qui rapporte que plus de femmes $(55 \%)$ que d'hommes ( $34 \%$ ) prennent des médicaments.

Parmi les consommatrices de drogues illicites, $9,1 \%$ ont fait usage de marijuana et d'autres drogues, contre $8,6 \%$ qui consommaient exclusivement de la marijuana. Il apparaît donc clairement que la drogue illicite la

6. Buveuses habituelles : personnes ayant consommé de l'alcool au moins une fois par mois. Source : Ministère de la Santé et des Services sociaux du Québec, 1988, p. 16.

7. Buveuses occasionnelles: personnes ayant consommé de l'alcool au cours de la dernière année, mais moins d'une fois par mois. Source: Ministère de la Santé et des Services sociaux du Québec, 1988, p. 16.

8. En termes de prévalence. 
plus utilisée est la marijuana. La proportion de Québécoises dont la consommation est problématique s'élève à $1,8 \%(4,4 \%$ pour les hommes $)$.

\section{B. Consommation de substances psycho-actives chez les adultes judiciarisés}

Aucune étude épidémiologique d'envergure ne s'était encore attardée à mesurer l'importance de la consommation de substances psycho-actives parmi les femmes incarcérées au Québec. Jusqu'à maintenant, il fallait consulter les enquêtes effectuées auprès des hommes détenus pour évaluer l'importance de l'usage de drogues chez les femmes judiciarisées au Québec.

Ainsi, par le biais d'une étude sur la prévalence des troubles mentaux dans les pénitenciers fédéraux du Québec, Hodgins et Côté (1990) révèlent que la moitié $(50,4 \%)$ des personnes incarcérées présentaient un problème d'abus ou de dépendance à l'alcool et que plus du tiers $(35,6 \%)$ des détenus éprouvaient des difficultés semblables face à au moins une drogue illicite. À partir des réponses de leurs sujets à une version française du Diagnostic Interview Schedule (DIS), les mêmes auteurs affiment que la polytoxicomanie constitue également un problème courant puisque, parmi ceux qui utilisent des drogues illicites de façon abusive, les trois quarts $(74,7 \%)$ font également un abus d'alcool.

Plus récemment, Forget (1990) a entrepris une étude auprès des détenus de la prison de Montréal. Un échantillon de 130 hommes a été constitué afin de déterminer, à l'aide d'un rapport auto-révélé, la prévalence de la consommation de substances psycho-actives parmi eux. Les résultats de l'enquête indiquent que $63,1 \%$ des personnes incarcérées ont rapporté avoir consommé du cannabis durant les 12 mois précédant l'incarcération, $56,9 \%$ ont pris de la cocaïne et $32,3 \%$ ont utilisé des médicaments. Au total, $63,1 \%$ ont fait usage d'au moins une drogue durant cette période (à l'exclusion du tabac et de l'alcool). Ce nombre diminue à $40 \%$ pour la consommation d'au moins une substance psycho-active au cours des 30 jours avant l'incarcération.

En ce qui concerne la dépendance face aux drogues illicites, $58,4 \%$ des détenus présentent un degré de toxicomanie allant de modéré à grave (tel que mesuré par une version française du Drug Abuse Screening TestDAST).

Les substances psycho-actives les plus couramment employées sont, en ordre d'importance, le cannabis, la cocaïne, les médicaments, les hallucinogènes, l'héroïne, les solvants et enfin les autres opiacés. Lorsque Forget (1990) compare ces résultats à ceux d'enquêtes récentes réalisées au Québec, il conclut qu'il existe une concentration beaucoup plus forte de consommateurs de drogues parmi les personnes incarcérées que dans la population générale. 
Plus spécifiquement chez les femmes, une étude du ministère du Solliciteur général du Québec (1988) estime que la toxicomanie s'avère très importante parmi les femmes détenues. Soixante-dix à $80 \%$ de la population féminine présenteraient des indices de toxicomanie et, de ce nombre, environ 35 à $40 \%$ d'entre elles seraient incarcérées pour un crime relatif à ce problème.

Dans un article paru en 1983, l'ancienne directrice (Valcourt, 1983) de la maison Thérèse-Casgrain, centre résidentiel communautaire qui accueille des femmes des prisons Tanguay et Kingston, établit que sur les 42 résidentes admises depuis l'ouverture, soit 1980 , seulement 9 n'ont pas présenté de problèmes de consommation de drogues pendant leur séjour.

Biron et Savard (1986) ont effectué une étude auprès de Québécoises ayant commis des délits graves. Ces femmes se trouvaient soit en libération conditionnelle $(n=9)$ soit en institution fermée $(n=8)$ ou ouverte $(n=2)$. La collecte de données s'est faite par entrevues suivant une méthode qualitative. Les résultats de la recherche permettent de constater que le choix des substances dépend en grande partie de leur disponibilité et de leur accessibilité. Les auteurs constatent qu'à la fin de leur adolescence, la majorité de ces femmes luttaient contre de sérieux problèmes de consommation. De plus, près de la moitié des sujets sont devenues dépendantes aux drogues dans un intervalle de 2 à 3 ans. La vie de ces femmes était centrée sur l'obtention de gains rapides en vue de pourvoir à leur ration de drogue. Cet assujettissement semble les avoir précipitées dans un environnement criminel et avoir augmenté leurs risques de commettre des délits. Ces femmes qui désiraient une accessibilité rapide aux produits sont devenues les victimes de leur compagnon de vie ou de revendeurs de drogues («pushers»). Ceux-ci en sont venus à cesser de fournir gratuitement te produit et ont exigé des «faveurs" afin de rembourser les dettes contractées. C'est ainsi qu'elles en venaient à travailler comme serveuses dans un bar, danseuses ou prostituées. Elles prenaient également part au trafic et à la vente de drogues. Lorsqu'une de ces activités ne suffisait pas à financer leur consommation, elles en pratiquaient plus d'une à la fois.

Il se dégage de cette recension qu'il existe peu d'études approfondies témoignant de l'ampleur du problème de la toxicomanie parmi la population féminine en détention. En conséquence, l'objectif de la présente analyse consiste à combler cette lacune.

\section{MÉTHODE}

\section{A. Sujets}

L'échantillon de cette étude est constitué de personnes provenant des deux prisons et des deux maisons de transition pour femmes au Québec. 
Tableau 1

Distribution des réponses selon le type d'infractions commises*

\begin{tabular}{|l|rr|rr|rc|}
\hline \multirow{2}{*}{$\begin{array}{l}\text { Catégories } \\
\text { d'infractions }\end{array}$} & \multicolumn{2}{|c|}{ Montréal } & \multicolumn{2}{|c|}{ Québec } & \multicolumn{2}{c|}{ Total } \\
& $\mathbf{n}$ & $\%$ & $\mathbf{n}$ & $\%$ & $\mathbf{n}$ & $\%$ \\
\hline Vol simple & 17 & 27,9 & 13 & 41,9 & 30 & 32,6 \\
Trafic de drogues & 18 & 29,5 & 5 & 16,1 & 23 & 25,0 \\
Possession de drogues ${ }^{1}$ & 17 & 27,9 & 4 & 12,9 & 21 & 22,8 \\
Fraude & 11 & 18,0 & 9 & 29,0 & 20 & 21,7 \\
Voies de fait & 9 & 14,8 & 4 & 12,9 & 13 & 14,1 \\
Vol qualifién & 10 & 16,4 & 3 & 9,7 & 13 & 14,1 \\
Possession d'arme & 11 & 18,0 & 2 & 6,5 & 13 & 14,1 \\
Prostitution & 8 & 13,1 & 1 & 3,2 & 9 & 9,8 \\
Incendie criminel & 2 & 3,3 & 4 & 12,9 & 6 & 6,5 \\
Introduction par effraction & 2 & 3,3 & 2 & 6,5 & 4 & 4,3 \\
Meurtre & 2 & 3,3 & 2 & 6,5 & 4 & 4,3 \\
Menace/harcèlement & 2 & 3,3 & 1 & 3,2 & 3 & 3,3 \\
Séquestration & 2 & 3,3 & 1 & 3,2 & 3 & 3,3 \\
Homicide & 2 & 3,3 & 0 & 0,0 & 2 & 2,2 \\
Vandalisme & 1 & 1,6 & 1 & 3,2 & 2 & 2,2 \\
Enlèvement & 1 & 1,6 & 0 & 0,0 & 1 & 1,1 \\
\hline
\end{tabular}

* Plus d'une réponse pouvait être mentionnée par les personnes interrogées.

1. Cette catégorie inclut le délit de possession de drogues (article 3) et la possession en vue d'en faire le trafic (article 4 (2)) du Code criminel canadien.

Toutes les femmes disponibles ont été sollicitées pour participer à l'enquête. Néanmoins, on a exclu: 1) les personnes unilingues anglaises ne pouvant lire ou comprendre le questionnaire rédigé en français; 2 ) les femmes faisant partie de l'aile psychiatrique ou clinique; 3) les femmes profitant de programmes à l'extérieur de la prison (bien sûr, celles qui se retrouvaient dans les deux maisons de transition faisaient partie de l'étude); 4) les femmes n'ayant pas le statut de citoyennes canadiennes.

Au moment de la visite des chercheurs, la population totale disponible de ces centres se chiffrait à 132 femmes. De ce nombre, 94 ont accepté de répondre au questionnaire. (Cependant, quatre d'entre elles ne l'ont pas totalement complété.) La présente étude rejoint donc $71,2 \%$ des Québécoises détenues.

Parmi les 94 femmes de l'échantillon, $18(19,1 \%)$ étaient en attente de procès, $56(56,9 \%)$ étaient détenues et 20 femmes $(21,3 \%)$ étaient en maison de transition. 
Soixante et une femmes (61) provenaient de la région de Montréal $(64,9 \%)$, les autres de la région de Québec et de sa périphérie.

L'échantillon est composé en majorité de femmes purgeant une sentence provinciale de deux ans moins un jour $(73,4 \% ; n=69)$.

Dans l'ensemble, les crimes commis s'avèrent de type non-violent. Les vols simples $(32,6 \%$ des personnes interrogées), le trafic de stupéfiants $(25,0 \%)$, la possession de drogues $(22,8 \%)$ et la fraude $(21,7 \%)$ constituent les principaux délits des contrevenantes de cette étude (tableau 1).

\section{B. Instruments de cueillette des données}

Les données furent recueillies à l'aide de questions fermées. Le premier questionnaire comprenait 314 variables et se divisait en neuf sections : 1) informations de base ; 2) renseignements socio-démographiques; 3) relations familiales/sociales; 4) milieu de vie; 5) statut légal ; 6) consommation de substances psycho-actives ; 7) habitudes de consommation ; 8) rôle joué par l'alcool/les drogues dans l'incarcération; 9) désintoxication et traitement. Ce questionnaire a été tiré de l'étude sur la consommation des jeunes en centre d'accueil effectuée par Brochu et Douyon (1990) et adapté à la situation des femmes contrevenantes.

Deux autres questionnaires élaborés à l'Addiction Research Foundation de Toronto ont également été utilisés : le premier, l'Alcohol Dependence Scale (ADS), a êté élaboré et validé par Skinner (1982) afin d'évaluer le niveau d'accoutumance à l'alcool; tandis que le second, le Drug Abuse Screening Test (DAST), élaboré et validé par Skinner et Horn (1984), établit le taux de dépendance aux drogues.

\section{i Alcohol Dependence Scale (ADS)}

L'Alcohol Dependence Scale (ADS) a été conçu afin de fournir un indice rapide du niveau de dépendance à l'alcool. En ce qui concerne les qualités psychométriques de l'ADS, Skinner and Allen (1982) rapportent un niveau de consistance interne s'élevant à ,92. Une analyse du test-retest à un intervalle d'une semaine s'élève également à ,92. En ce qui concerne la validité concurrente, l'ADS présente une corrélation de ,67 avec l'Alcohol Use Inventory (Kivlahan, Sher and Donovan, 1989).

\section{ii Drug Abuse Screening Test (DAST)}

Le Drug Abuse Screening Test (DAST) (Skinner, 1982) a été conçu selon des principes semblables à l'ADS afin de fournir rapidement un indice

9. Tout au long de cette recherche, les termes «dépendance» et «accoutumance " seront considérés dans ce texte comme des synonymes. 
sur le degré de dépendance aux drogues. Les résultats au DAST sont en corrélation significative avec l'abus ou la dépendance aux drogues tels que mesurés par le DSM III $(r=0,85)$. La validité du questionnaire est élevée, soit un alpha de 0,92 (Skinner, 1982).

Les qualités psychométriques d'une adaptation française de ces deux instruments appliqués à des détenus ont été vérifiées par une équipe de chercheurs du Service correctionnel canadien (Weeekes et coll., 1994). Leurs résultats se comparent favorablement aux qualités métrologiques de l'instrument original.

\section{RÉSULTATS}

\section{A. Consommation de substances psycho-actives au cours de la vie}

La prévalence d'usage (expérimentale ou plus) au cours de la vie de substances psycho-actives parmi la population féminine judiciarisée se situe à $94,7 \%$ si l'on considère le nombre de détenues qui ont déjà fait l'essai d'au moins une drogue (y compris l'alcool). La comparaison de la consommation à vie à celle des 30 jours précédant l'incarcération (voir le tableau 2) indique que, dans bien des cas, il s'agit d'une consommation expérimentale ou irrégulière de produits plutôt que d'un usage régulier.

L'alcool constitue bien sûr le produit le plus expérimenté par les femmes rencontrées $(89,4 \%)$. La cocaïne $(71,3 \%)$ et le cannabis $(68,1 \%)$ sont les deux produits illicites les plus utilisés de façon expérimentale. Un fait surprend cependant : plus de femmes rapportent avoir déjà utilisé de la cocaïne que du cannabis. Les ballucinogènes $(55,3 \%)$ précèdent de peu les médicaments $(51,1 \%)$. Les opiacés, autres que l'héroïne $(28,7 \%)$, l'héroïne $(26,6 \%)$, les substances volatiles $(17,0 \%)$, et la méthadone $(13,8 \%)$ demeurent des produits relativement peu essayés par les femmes interrogées. On remarque de plus que $10,6 \%$ des contrevenantes ont mentionné n'avoir jamais consommé d'alcool. Ce pourcentage est à peu près analogue à celui qui ressort de l'enquête de Santé Bien-Être social Canada (voir Brochu, Mercier et Ouimet, 1991) qui rapporte que $9,4 \%$ des femmes canadiennes n'ont jamais consommé d'alcool. On aurait pu s'attendre à ce que le taux d'abstinence à vie soit beaucoup plus petit chez les détenues.

Les entrevues effectuées ont permis de constater que, de façon générale, le crack demeure un produit peu répandu parmi la population carcérale féminine. Selon les affirmations des personnes rencontrées, seulement $14,9 \%$ en ont fait l'expérimentation. Les commentaires négatifs formulés par les détenues à l'égard de ce produit peuvent laisser croire que les campagnes de prévention ont porté fruit parmi celles qui font l'expérience et même l'usage régulier de drogues. Il ne faut toutefois pas sauter aux conclusions trop rapidement, puisque plusieurs de ces détenues affirment 
consommer de la freebase. Un questionnement relatif à sa méthode de préparation nous laisse cependant croire qu'elle s'apparente surtout au crack. Il est donc probable que la perception négative associée au crack fasse en sorte que ce type de consommation soit sous-évaluée et donc plus étendue qu'on ne le rapporte.

Tableau 2

Répartition des personnes interrogées selon les produits déjà utilisés

\begin{tabular}{|l|rr|rr|rr|}
\hline \multirow{2}{*}{ Produits* } & \multicolumn{2}{|c|}{ À vie } & \multicolumn{2}{c|}{12 mois } & \multicolumn{2}{c|}{30 jours } \\
& n & \% & \multicolumn{1}{c|}{ n } & \% & n & \% \\
\hline Alcool & 84 & 89,4 & 65 & 69,1 & 58 & 61,7 \\
Cocaïne & 67 & 71,3 & 55 & 58,5 & 48 & 51,1 \\
Cannabis & 64 & 68,1 & 44 & 46,8 & 39 & 41,5 \\
Hallucinogènes & 52 & 55,3 & 22 & 23,4 & 16 & 17,0 \\
Médicaments & 48 & 51,1 & 37 & 39,4 & 30 & 31,9 \\
Autres opiacés** & 27 & 28,7 & 12 & 12,8 & 9 & 9,6 \\
Hêroïne & 25 & 26,6 & 16 & 17,0 & 14 & 14,9 \\
Substances volatiles & 16 & 17,0 & 5 & 5,3 & 4 & 4,3 \\
Méthadone & 13 & 13,8 & 8 & 8,5 & 4 & 4,3 \\
\hline
\end{tabular}

* Plus d'un produit pouvaient être énumérés par les répondantes.

** Autres opiacés = opium, morphine, codéine, démérol.

\section{B. Âge d'expérimentation des substances psycho-actives}

Dans l'ensemble, l'expérimentation des drogues s'étend surtout entre 11 et 21 ans $(\chi=18,6 ; \sigma=5,3)$. À l'intérieur de cet écart, plus les femmes avancent en âge, plus il s'avère que la consommation de substances psychoactives se déplace vers des drogues dites dures (telles les hallucinogènes, la cocaïne et l'héroüne). Par la suite, l'expérimentation de nouveaux produits est plus rare et on observe une certaine stabilité à l'intérieur des drogues déjà expérimentées. Ces résultats confirment ceux rapportés par Biron et Savard (1986).

Un fait attire l'attention et se démarque de l'ensemble du profil des femmes à l'étude. On remarque que certaines contrevenantes $(n=6)$ ont consommé des substances psycho-actives avant l'âge de 11 ans. Brochu et Douyon (1990) avaient également noté l'initiation précoce aux drogues de certaines personnes de leur échantillon de jeunes placés en centre d'accueil. Ces auteurs expliquaient cette situation par des facteurs environnementaux pouvant jouer un rôle dans cet apprentissage. 
Il est intéressant de noter que l'âge moyen du premier délit révélé ${ }^{10} \mathrm{par}$ les contrevenantes s'élève à 22,2 ans (médiane $=18$ ans), alors que les premières expérimentations d'alcool et d'autres drogues se situent respectivement en moyenne à 16 ans (médiane = 15 ans) et à 19 ans (médiane 17,4). Ainsi, la consommation de substances psycho-actives a lieu avant la conduite délinquante. Toutefois, entre cette consommation et le début de la délinquance, il s'écoule un laps de temps relativement court. Cette donnée s'avère importante car, trop souvent, les études de prévalence sont utilisées pour soutenir l'hypothèse d'un lien causal entre la consommation de drogues et la délinquance des personnes judiciarisées. Dans ce cas-ci, cette hypothèse ne peut être soutenue pour la majorité des sujets, bien qu'un certain nombre d'entre elles aient débuté leur délinquance après leur initiation à la drogue.

\section{Consommation de substances psycho-actives au cours de l'année précédant l'incarcération}

À la question concernant l'usage de substances psycho-actives durant les douze mois précédant l'incarcération, l'alcool apparaît à nouveau, et ce sans surprise, comme le produit le plus utilisé par les femmes de l'échantilIon $(69,1 \%)$. Parmi les drogues illicites, la cocaïne $(58,5 \%)$ et le cannabis $(46,8 \%)$ demeurent des produits hautement répandus, avec, encore une fois une prédominance de la cocaïne sur le cannabis. Les médicaments nonprescrits $(39,4 \%)$ représentent également des produits relativement populaires. À l'inverse, l'hérö̈ne $(17,0 \%)$, les autres opiacés $(12,8 \%)$ ainsi que les solvants volatils $(5,3 \%)$ ne sont consommés que par un petit groupe de femmes contrevenantes (tableau 2). Dans l'ensemble, $84,0 \%$ des contrevenantes ont consommé au moins une substance psycho-active au cours des douze mois précédant l'incarcération. Parmi elles, $73,4 \%$ ont révélé avoir consommé au moins une drogue illicite et $69,1 \%$ de l'alcool. Par ailleurs, les résultats démontrent que, pour la même période à l'étude, $64,9 \%$ des contrevenantes étaient des polyconsommatrices. À l'inverse, seulement $19,1 \%$ des personnes rencontrées consommaient un seul produit. En tout, $12,8 \%$ des répondantes ont avoué ne pas avoir consommé de substances psycho-actives durant cette période (sont incluses les anciennes consommatrices et les abstinentes) alors que 3,2\% des sujets de l'échantillon n'ont pas fourni d'information.

10. Le terme « délit révélé » renvoie ici à un acte commis sans être nécessairement connu du système pénal; c'est ce qu'on appelle le chiffre noir de la criminalité. 


\section{Consommation de substances psycho-actives au cours du mois précédant l'incarcération}

Encore une fois, une majorité de sujets $(61,7 \%)$ ont consommé de l'alcool au cours des 30 jours précédant leur incarcération (voir le tableau 2). L'étude des données présentées au tableau 2 révèle également que les deux autres substances les plus importantes sont dans l'ordre la cocaïne $(51 \%$ d'usagères) et le cannabis ( $42 \%$ d'utilisatrices).

L'analyse plus détaillée des statistiques descriptives de la consommation du mois précédant l'incarcération permet de noter l'existence de deux principaux modèles de consommation (voir le tableau 3). Il existe d'une part des produits tels l'héroïne, la cocaïne, les médicaments et le cannabis dont on fait un usage quotidien (mode $=30$; médiane $>15$ ) et, par ailleurs, d'autres produits tels les hallucinogènes, la méthadone ou les substances volatiles dont on fait une consommation occasionnelle (mode $<3$; médiane $<15$ ).

Tableau 3

Fréquence de consommation de droguess au cours des 30 jours précédant l'incarcération

\begin{tabular}{|l|rr|rr|rrrr|}
\hline Substances* & \multicolumn{2}{|c|}{$\begin{array}{c}\text { Pendant 1 à } \\
\text { 5 jours }\end{array}$} & \multicolumn{2}{|c|}{$\begin{array}{c}\text { Pendant 6 à } \\
\text { 19 jours }\end{array}$} & \multicolumn{4}{|c|}{ Pendant 20 jours } \\
& ou plus \\
& n & $\%$ & \multicolumn{1}{c|}{ n } & \% & n & $\%$ & $\chi$ & $\sigma$ \\
\hline Cannabis & 14 & 14,9 & 8 & 8,7 & 16 & 17,1 & 15,9 & 12,4 \\
Cocainne & 14 & 14,9 & 10 & 10,8 & 24 & 25,6 & 18,6 & 12,0 \\
Médicaments & 5 & 5,4 & 5 & 5,4 & 14 & 14,9 & 20,0 & 11,8 \\
Hallucinogènes & 9 & 9,6 & 3 & 3,2 & 1 & 1,1 & 12,3 & 23,2 \\
Subst. volatiles & 2 & 2,1 & 1 & 1,1 & 1 & 1,1 & 13,3 & 13,0 \\
Héroine & 2 & 2,1 & 2 & 2,2 & 9 & 9,6 & 22,9 & 11,5 \\
Méthadone & 2 & 2,1 & 1 & 1,1 & 0 & 0,0 & 2,7 & 2,9 \\
\hline
\end{tabular}

1. La fréquence de consommation n'a pas été demandée pour l'alcool.

* Plus d'un produit peut être rapporté par les personnes interrogées.

Cette dernière constatation nous amène à nous interroger sur la dépendance de ces femmes. En effet, l'usage quotidien d'héroïne ou de cocaïne a sûrement une signification psychologique et physique différente d'une consommation occasionnelle d'hallucinogènes. Dans une optique de prestations de service, une meilleure connaissance des niveaux de dépendance à l'alcool ou aux autres drogues permettrait d'instaurer des programmes répondant davantage aux besoins existants (par exemple : sessions de sensibilisation aux drogues; prévention primaire ciblée; prévention secondaire 
rapide ; programmes pré-libératoires préparant à l'entrée en traitement, programmes de traitement).

\section{E. Dépendance à l'alcool ou aux autres drogues}

Le questionnaire ADS sur la consommation d'alcool (Skinner et Horn, 1984) permet de classer les consommatrices en fonction de leur niveau de dépendance. Les résultats au test de l'ADS démontrent qu'un peu moins de la moitié des répondantes $(44,8 \%)$ enregistrent un taux de dépendance « très limité » à l'alcool (voir tableau 4). Selon les auteurs de l'ADS (Skinner et Horn, 1984), cette forme de dépendance s'avère probablement plus psychologique que physique. Près de $8,0 \%$ des répondantes présentent une dépendance "modérée » à l'alcool. Les personnes ayant ce degré de dépendance éprouvent, toujours selon Skinner et Horn (1984), des problèmes psycho-sociaux liés à leur consommation. Les auteurs mentionnent que la dépendance psychologique peut être la caractéristique principale de cette assuétude à l'alcool, mais on découvre parfois chez les sujets des signes de dépendance physique ainsi que des symptômes de sevrage. On compte $5,4 \%$ de répondantes qui présentent une dépendance «élevée » à l'alcool. Ce niveau est fortement associé à la probabilité que le sujet souffre d'une dépendance physique. Enfïn, 1,1\% des consommatrices présentent un degré "grave » de dépendance à l'alcool. Ceci signifie une dépendance physique évidente accompagnée de la présence de désordres physiques sérieux liés à cette consommation, tels les maladies du foie (Skinner et Horn, 1984).

Tableau 4

Répartition des contrevenantes selon le niveau de dépendance à l'alcool (ADS) ou aux autres drogues (DAST)

\begin{tabular}{|l|rr|rc|}
\hline Degrés de & \multicolumn{3}{|c|}{ ADS } & \multicolumn{2}{c|}{ DAST } \\
dépendance & $\mathbf{n}$ & $\%$ & \multicolumn{1}{c|}{$\mathbf{n}$} & $\%$ \\
\hline Nul & 12 & 12,8 & 0 & 00,0 \\
Très limité & 42 & 44,8 & 12 & 12,8 \\
Modéré & 7 & 7,5 & 15 & 16,0 \\
Élevé & 5 & 5,4 & 26 & 27,6 \\
Grave & 1 & 1,1 & 14 & 14,9 \\
Ne s'applique pas* & 24 & $\mathbf{2 5 , 5}$ & 24 & 25,5 \\
Pas d'information & 3 & $\mathbf{3 , 2}$ & 3 & 3,2 \\
\hline Total & $\mathbf{9 4}$ & $\mathbf{1 0 0}$ & $\mathbf{9 4}$ & $\mathbf{1 0 0}$ \\
\hline
\end{tabular}

* La catégorie «ne s'applique pas " comprend les contrevenantes ayant révélé ne pas avoir consommé aux cours des 12 derniers mois précédant l'incarcération.

Il faut cependant demeurer prudent concernant les niveaux de dépendance suggérés par le test de l'ADS, car il ne tient pas compte de la dépendance 
aux autres drogues chez les polytoxicomanes. Ce qui a pour effet de minimiser la portée réelle de la dépendance à l'ensemble des substances psychoactives.

Le DAST (Skinner, 1982), pour sa part, permet d'attribuer un degré de dépendance aux autres drogues (y compris les médicaments non-prescrits) à chacune des répondantes. Comme l'illustre le tableau 4, 12,8\% des contrevenantes de l'échantillon présentent un degré d'accoutumance aux drogues « très limité ». Les données recueillies démontrent, par ailleurs, que le degré de dépendance aux drogues est «modéré » chez $16,0 \%$ des contrevenantes, "élevé » chez $27,6 \%$ et "grave" chez $14,9 \%$. À l'inverse, $25,5 \%$ des personnes interrogées n'ont aucune dépendance associée à la consommation de stupéfiants. Il faut souligner que ce dernier groupe de répondantes ont affirmé ne pas avoir consommé au cours des 12 mois précédant l'incarcération car la construction du test est telle qu'une seule consommation de drogues au cours des 12 mois précédant la passation du questionnaire place la personne à un niveau de dépendance très limité. La comparaison de l'ADS et du DAST montre bien le double standard existant entre la simple consommation de l'alcool et la simple consommation des autres drogues. Il devient alors impossible de comparer les résultats des deux tests.

\section{F. La volonté des détenues à s'inscrire à un traitement de la toxicomanie}

Après avoir constaté l'ampleur de la problématique toxicomane chez les détenues, il est intéressant de mieux connaître leur motivation à entreprendre une démarche de changement. Selon leur propre déclaration, plus d'une consommatrice ${ }^{11}$ sur deux expriment le désir de suivre un traitement de la toxicomanie (41/75). Les motifs évoques pour s'inscrire dans un tel processus de changement sont multiples et, bien entendu, plus d'une raison peuvent être considérées par une même personne. Parmi celles qui ressortent avec force, notons le désir d'éviter un retour éventuel en prison (34/41), la recherche de l'abstinence (32/41), la volonté de mieux s'occuper de leur enfant (24/41), l'intention de ne plus commettre de nouveaux délits (24/41) et, finalement, la décision de résoudre leurs problèmes financiers (23/41).

Considérant ces résultats, il fut décidé d'interroger 50 intervenants (44 femmes et 6 hommes) travaillant dans ces centres de détention de façon à recueillir leurs propos concernant l'intervention qui devrait être offerte aux femmes détenues aux prises avec des problèmes de toxicomanie. D'entrée de jeu, les intervenants ont exprimé leur préoccupation face à la consommation de drogues des contrevenantes. À l'instar des intervenants judiciaires de l'Ontario interviewés par Lightfoot et coll. (1985), la vaste majorité des

11. Consommatrice : personne qui a consomme une drogue au cours des 12 mois précédant son incarcération. 
personnes interrogées $(80 \%)$ lors de la présente étude affirment qu'il est nécessaire d'offrir un continuum de services à partir de l'incarcération jusqu'à la remise en liberté. Toutefois, pour les intervenants interrogés, les programmes spécialisés offerts en communauté viendraient compléter une démarche plus générale de réadaptation amorcée lors de la détention. Plus spécifiquement, durant l'incarcération, les intervenants nous informent qu'ils offrent des services d'écoute, des programmes d'éducation de groupe et des thérapies individuelles à l'ensemble des détenues et, bien entendu, aux toxicomanes qui en font la demande. Certains suggèrent qu'il serait bon que les institutions se dotent d'un plan d'intervention plus spécifique en matière de toxicomanie de façon à que que tous les intervenants suivent les mêmes lignes directrices et appuient leurs actions sur des pratiques précises et fiables.

Le renvoi à des programmes spécialisés lors de la libération devrait, selon les intervenants interrogés, s'appuyer sur le profil de motivation de même que sur la demande d'aide formulée par la contrevenante. Quoique les intervenants se disent généralement satisfaits de la collaboration avec les agences de traitement de la toxicomanie de leur communauté environnante, certains nous informent que les critères d'admission de quelques centres de traitement constituent parfois des barrières difficilement surmontables par les femmes contrevenantes soumises aux exigences du système pénal. D'autres intervenants se disent mal à l'aise face à la référence de leur clientes vers des centres de traitement dont Ja programmation leur est souvent inconnue et avec lesquels il y a absence de concertation.

Une étude présentement en cours au centre de détention Tangay, utilisant l'Indice de gravité d'une toxicomanie (IGT) (Bergeron et coll.,) une version française de l'Addiction Severity Index (ASI) (Mc Lellan et coll., 1980) permettra de mieux cerner la dépendance des femmes détenues et leurs besoins de traitement en toxicomanie.

\section{CONCLUSION}

Il apparaît donc clairement que la consommation de substances psychoactives chez les contrevenantes s'avère supérieure à celle relevée chez l'ensemble des femmes québécoises. Néanmoins, il faut faire preuve de prudence dans ce type de rapprochement, car les deux études sont sensiblement différentes sur le plan des méthodes utilisées.

En comparant les résultats de la présente étude à l'enquête de Santé et Bien-être social Canada (voir Brochu, Mercier et Ouimet, 1991), la consommation à vie de cannabis et de cocaïne est nettement plus importante chez les femmes que nous avons interrogées que parmi l'ensemble de la population féminine canadienne (respectivement $68,1 \%$ et $17,7 \%$ pour le cannabis ; $71,3 \%$ et $2,7 \%$ pour la cocaïne). 
Lorsque l'on s'attarde à la consommation de substances psycho-actives au cours d'une période de 12 mois, l'écart apparaît encore plus considérable. Santé et Bien-être social Canada (voir Brochu, Mercier et Ouimet; 1991) révèle que $4,1 \%$ des Canadiennes ont consommé du cannabis et $0,8 \%$ de la cocaïne. Chez les Québécoises (ministère de la Santé et des services sociaux du Québec; 1988), 9,1\% ont révélé avoir consommé du cannabis. La donnée québécoise montre une plus grande proportion de consommatrices de cannabis qu'à l'échelle nationale. Malgré les différences sur le plan des méthodes utilisées, on constate que la consommation de drogues illicites, telles la cocaïne et le cannabis, est beaucoup plus élevée parmi les contrevenantes de l'étude comparativement à la population féminine canadienne. De plus, la cocaïne arrive bon premier avant le cannabis au sein de la population carcérale féminine.

À l'inverse, il apparaît que la consommation d'alcool est un peu moins fréquente chez les contrevenantes de l'étude $(69,1 \%)$ que chez les femmes québécoises $(74,8 \%$ ) (ministère de la Santé et des services sociaux du Québec, 1988 ) et canadiennes $(71,8 \%$ ) (voir Brochu, Mercier et Ouimet, 1991).

Pour sa part, l'étude du ministère de la Santé et des Services sociaux du Québec (1988) a établi le degré de dépendance à l'alcool des femmes québécoises à l'aide des critères du DSM-III. Les résultats démontrent que seulement $2,8 \%$ des répondantes enregistrent un tel problème d'alcool. Malgré les différences sur le plan des méthodes utilisées, on peut établir clairement que le problème de dépendance à l'alcool s'avère beaucoup plus élevé chez les femmes de cette étude $(13,8 \%$ ayant une dépendance de modérée à grave) que chez les femmes de la province de Québec.

De son côté, l'étude du ministère de la Santé et des Services sociaux du Québec (1988) établit le degré de dépendance aux drogues à $1,8 \%$ parmi les femmes québécoises. À l'opposé, une étude du ministère du Solliciteur général du Québec (1988) établissait que près de $70 \%$ des femmes incarcérées auraient développé une dépendance aux drogues. Les résultats de la présente étude $(58,5 \%)$, même s'ils ne sont pas aussi élevés, indiquent bien que les contrevenantes présentent une dépendance aux drogues de beaucoup supérieure à celle de l'ensemble des Québécoises.

En ce qui concerne la consommation de médicaments, il s'avère difficile de comparer les résultats de la présente étude à ceux du ministère de la Santé et des Services sociaux du Québec (1988) ou aux résultats de l'enquête nationale réalisée par Santé et Bien-être social Canada puisque ces deux dernières études comptabilisaient à la fois les médicaments prescrits et non-prescrits.

En somme, la prévalence de consommation de substances psychoactives chez les femmes détenues au Québec s'avère très importante. En 
fait, bon nombre d'entre elles se décrivent comme des polyconsommatrices. Elles font un usage régulier de drogues fortes (particulièrement la cocaïne) qui entraîne pour plusieurs d'entre elles une dépendance.

La majorité des prisons pour femmes en Amérique du Nord sont désavantagées en termes de services en comparaison des centres de détention pour hommes. Malgré Ja forte prévalence de consommation et de dépendance à la drogue de la part des femmes détenues, au Québec comme dans beaucoup d'autres pays du monde, les femmes n'ont pas accès aux types de programmes thérapeutiques offerts à leur contrepartie masculine. Durant nos visites relatives à la présente étude, les seuls services spécialisés en toxicomanies disponibles pour les détenues étaient les mouvements d'entraide tels les Alcooliques anonymes (A.A.), les Cocaïnomanes Anonymes (C.A.), ainsi que les Narcotiques anonymes (N.A.). Bien que ces groupes d'entraide promeuvent un style de vie sans drogue, il n'en demeure pas moins qu'ils ne peuvent répondre à toutes les demandes de réadaptation des femmes détenues (Lipton, Falking et Wexler, 1992). Même si la majorité des professionnels travaillant auprès des détenus croit que ces groupes d'entraide apportent une contribution significative, ils ont l'impression que ces types de services ne sont pas suffisants et devraient être combinés à des programmes plus intensifs (Service correctionel du Canada, 1991). Le petit nombre de femmes incarcérées étant souvent évoqué pour justifier l'inaction des autorités, le recours à des formes d'intervention moins classiques pourrait pallier cette situation. On peut alors penser au développement de liens plus étroits avec les agences de traitement établies dans la communauté. L'embauche d'intervenants spécialisés en toxicomanie pourrait également permettre la mise sur pied de sessions de sensibilisation, de programmes préventifs ou de modules pré-libératoires visant à faciliter l'insertion des toxicomanes dans un groupe de thérapie lors de leur libération conditionnelle. Parallèlement, il faudrait réfléchir sur la possibilité d'offrir des services d'intervention minimale en réadaptation durant la détention (Peters et May, 1992).

Les informations recueillies concernant la consommation de substances psycho-actives chez les détenues ne doivent toutefois pas être généralisées à l'ensemble des délinquantes. En effet, les détenues ne sont habituellement pas représentatives de l'ensemble des contrevenantes en ce qui concerne leur consommation de drogues. On soupçonne que les femmes incarcérées consomment davantage que celles qui ne se font pas appréhender. Le besoin de consommer est parfois considéré comme un motif de perpétration d'actes criminels (besoin d'argent pour se procurer de la drogue), mais l'intoxication peut également constituer la raison principale de l'arrestation lors du délit (l'intoxication fait parfois disparaître les craintes et facilite ainsi l'implication dans un délit risqué; de même, l'intoxication diminue les réflexes et la capacité de réaction, créant ainsi une 
situation favorable pour les policiers chargés de la poursuite des contrevenantes ${ }^{12}$ ). Tout ceci sans parler de la délinquance reliée aux lois canadiennes sur les drogues (exemple: possession de stupéfiants), laquelle favorise une sur-représentation des contrevenantes consommatrices parmi l'ensemble des détenues.

Enfin, un dernier facteur peut contribuer à expliquer le nombre élevé de consommatrices et de toxicomanes parmi les détenues : l'inadaptation sociale profonde et prolongée de certaines consommatrices fait en sorte que celles-ci se retrouvent davantage dans des milieux encadrants comme la prison. Il s'agit pourtant d'une minorité de consommatrices à partir de laquelle on a parfois tendance à généraliser à l'ensemble des usagers de drogues. Sont-elles inadaptées à cause de leur consommation? Comment étaient-elles avant leur consommation? Que seraient-elles sans la drogue? Il est présentement impossible de répondre à ces questions, mais un fait demeure, ces personnes ne semblent pas avoir été happées, malgré leur volonté, dans une carrière criminelle uniquement à cause de leur consommation. On se détache donc ici d'une vision positiviste voulant que la drogue conduise au crime. La réalité de ces personnes est beaucoup plus complexe que cette phrase cliché ne peut le dire.

L'information fournie dans la présente étude démontre qu'une attention particulière doit être accordée à la problématique de consommation de substances psycho-actives parmi les contrevenantes incarcérées. Les résultats soulignent l'ampleur du problème dans le milieu carcéral et le besoin d'une intervention coordonnée. Il est plus que temps de mettre en place un plan d'action concerté dans le domaine de la toxicomanie pour les femmes judiciarisées. Ce plan d'action devrait comprendre la coordination des efforts dispensés, la formation des intervenants impliqués, la diversification des interventions fournies ainsi que l'évaluation de ces interventions.

Par ailleurs, l'étude nous a laissé l'impression que la toxicomanie ne constitue bien souvent qu'une des manifestations de l'inadaptation sociale profonde dont souffrent ces femmes. Une étude en cours pourra mieux nous renseigner sur le sujet. Néanmoins, on est en droit de croire que l'intervention auprès de ces femmes ne doit pas se limiter uniquement aux problèmes de toxicomanie.

12. Il en est de même pour les hommes. 


\section{BIBLIOGRAPHIE}

BERGERON, J., LANDRY, M., ISHAK, A., VEAUGEOIS, P. et TRÉPANIER, M. (1992), Validation d'un instrument d'évaluation de la gravité des problèmes reliés à la consommation de drogues et d'alcool. L'indice de gravité d'une toxicomanie (IGT), Montréal, RISQ.

BIRON, L. et SAVARD, C. (1986), Les Femmes auteures de délits graves, Ottawa, Solliciteur général du Canada, $\mathrm{n}^{\circ}$ 1986-16.

BROCHU, S. (1995), Drogues et criminalité : une relation complexe, Montréal, Presses de l'Université de Montréal.

BROCHU, S. et DOUYON, A. (1990), La Consommation de psychotropes chez les jeunes placés en Centre d'accueil, Montréal, Centre intemational de criminologie comparée.

BROCHU, S., MERCIER, C. et OUIMET, M. (1991), Testing the convergence hypothesis: gender differences in substance use for different age group, Montréal, International Centre for Comparative Criminology.

FORGET, C. (1990), La Consommation de substances psycho-actives chez les détenus du centre de détention de Montréal, Université de Montréal, mémoire de maîtrise inédit.

HODGINS, S. et CÔTÉ, G. (1990), « Prévalence des troubles mentaux chez les détenus des pénitenciers du Québec ", Santé mentale au Canada, vol. 38, pp. 1-5.

KIVLAHAN, D. R., SHER, K. J., et DONOVAN, D. M. (1989), «The alcohol dependence scale : a validation study among inpatient alcoholics", Journal of studies on alcohol, vol. 50, pp. 170-175.

LIGHTFOOT, et coll. (1985), Phase III of a Four Phases Proposal to Develop and Evaluate Offenders Alcohol and Drug Abusers Treatment Program, Ottawa, Services correctionnels du Canada.

LIPTON, D. S., FALKIN, G. P., et WEXLER, H. K. (1992), « Correctional Drug Abuse Treatment in the United States: An Overview », in NIDA Research Monograph Series, Drug Abuse Treatment in Prisons and Jails, Rockville, U.S. Department of Health and Human Services, pp. 8-30.

McLELLAN, A. T., LUBORSKY, L., WOODY, C. E. et O'BRIEN, C. P. (1980), « The Addiction Severity Index », The Journal of Nervous and Mental Disease, vol, 168, pp. 26-33.

MINISTÈRE DE LA SANTÉ ET DES SERVICES SOCIAUX (1988), Et la santé, ça va?, rapport de l'Enquête Santé Québec 1987, tome 1 et 2, Québec, Gouvernement du Québec.

MINISTÈRE DU SOLLICITEUR GÉNÉRAL DU QUÉBEC (1988), La Toxicomanie en milieu correctionnel, Sainte-Foy, Services correctionnels, Direction de la participation communautaire.

PETERS, R. et MAY II, R. (1992), « Drug Treatment Services in Jails ", in NIDA Research Monograph Series, Drug Abuse Treatment in Prisons and Jails, Rockville, U.S. Department of Health and Human Services, pp. 38-50.

SANTÉ ET BIEN-ÊTRE SOCIAL CANADA (1988), Rapport technique : Enquête promotion Santé Canada, Ottawa, Ministère des Approvisionnements et Services Canada. 
SANTÉ ET BIEN-ÊTRE SOCIAL CANADA (1990), Enquête nationale sur l'alcool et les autres drogues. Points saillants, Ottawa, Ministère des Approvisionnements et Services Canada.

SERVICE CORRECTIONNEL DU CANADA (1991), Rapport du groupe d'étude sur la réduction de la toxicomanie, Ottawa, Service Correctionnel du Canada.

SKINNER, H. A. (1982), « Drug Abuse Screening Test (D.A.S.T.) », Addiction behavior, vol. 7, pp. 363-371.

SKINNER, H. A. et ALLEN, B. A. (1982), « Alcohol Dependence Syndrome : Measurement and Validation ", Journal of Abnormal Psychology, vol. 91, pp. 68-87.

SKINNER, H. A., HORN, J. L. (1984), Alcohol Dependence scale (A.D.S.). User's Guide, Toronto, Addiction Research Foundation, pp. 1-38.

VALCOURT, D. (1983), "La Maison Thérèse Casgrain, une ressource communautaire», Criminologie, vol. XVI, pp. 113-119.

WATTERS, J. K., REINARMAN, C. et FAGAN, J. (1985), "Causality, context, and contingency : relationships between drug abuse and delinquency ", Contemporary Drug Problem, vol. 12, pp. 351-373.

WEEKES, J. R., VANDEBURG, S. A. et MILSON, W. A. (1994), A Comparison of the French and English Versions of the Computerized Lifestyle Assessment Instrument, Ottawa, Service correctionnel du Canada.

WEINER, N. A. et WOLFGANG, M. V. (1990), Pathways to criminal violence, Newbury Park, Sage publications inc, $237 \mathrm{p}$.

WEISSMAN, J. C., MARR, S. W. et KATSAMPES, P. L. (1976), "Addiction and criminal behavior : a continuing examin of criminal addicts ", Journal of drug issues, vol. 6, pp. 153-165.

WEISSMAN, J. C. et NASH, G. (1978), «A guide to the treatment of drug addict criminality ", Journal of drug issues, vol. 8, pp. 113-122.

WELTE, J. W. et MILLER, B. A. (1987), «Alcohol use by violent and property offenders », Drug and alcohol dependence, vol. 19, pp. 313-324.

WHITE, H. R. (1990), "The drug-use-delinquency connection in adolescence», in R. WEISHEIT (dir.), Drugs, Crime and the Criminal Justice System, Cincinnati, Anderson, pp. 215-256.

WILLIAMS, T. M. et KORNBLUM, W. (1985), Growing up poor, Lexington, Mass., Lexington Books.

WISH, E. D. et GROPPER, B. A. (1989), «Drug testing by the criminal justice system" in M. TONRY et J. Q. WILSON (dir.), Drugs and crime, Chicago, The University of Chicago Press, pp. 321-391.

ZINBERG, N. E. (1984), Drug, set, and setting: The social bases of controlled drug use, New Haven, Conn., Yale University Press. 